Article

\title{
Complex Multi-System Integration Problems Associated with Titanium Metalworking and Manufacture: System of Systems Aproach-Part I
}

\author{
Adam Stroud ${ }^{1}$ and Atila Ertas ${ }^{2, * \mathbb{D}}$ \\ 1 Ellwood Texas Forge, Houston, TX 77045, USA; adamstroudphd@gmail.com \\ 2 Department of Mechanical Engineering, Texas Tech University, Lubbock, TX 79409, USA \\ * Correspondence: aertas@coe.ttu.edu; Tel.: +1-(806)-834-57788
}

Received: 11 January 2019; Accepted: 26 March 2019; Published: 9 April 2019

\begin{abstract}
Titanium has an excellent combination of properties that make it an attractive material for use in aerospace applications. The one area in which titanium is not aligned with customer needs is affordability. Components made from titanium are many times more expensive than those manufactured from other alloys. The supply chain of an extruded product is no exception. A breakthrough in extrusion cost reduction would enable wider adoption of titanium in many structural member applications. In an effort to accomplish any breakthrough in titanium component costs, the entire supply chain for manufacturing should be evaluated simultaneously. Due to the complex interaction of the many facets of the systems in a manufacturing supply chain, it is inferred that the supply chain in its entirety must be the focus of the design activity in order to be successful. Design improvements on a single facet of manufacture may have little to no effect on the manufacture of the component. If the improvement has a detrimental impact on another system in the supply chain, overall performance may be lowered. The use of a system of systems' (SoS) design approach was used due to its capability to address complex multi-system integration problems associated with titanium metalworking and manufacture.
\end{abstract}

Keywords: system of systems; complexity; multi-system integration; titanium metalworking and manufacture

\section{Introduction}

Although titanium is a modern metal, commercially available only since the 1950s, it was first discovered in 1791 and is the ninth-most rich element in the Earth and the seventh-most abundant metal. South Africa is currently the second largest producer of titanium minerals in the world, contributing 22 percent of the global output. Australia and South Africa together account for just over 50 percent of the total supply of titanium mineral concentrates [1].

Titanium has distinct physical and chemical properties-it is elastic and tough, has a high strength to weight ratio, hardly expands with increasing temperatures, and can withstand cold without becoming brittle. Gamma titanium aluminide intermetallics show an excellent strength-weight ratio, and corrosion resistance at high temperature [2]. Although Gamma titanium has promising application possibilities, drilling machinability studies are the main interest due to presented machinability problems [3]. In spite of the abundance and advantages of titanium metal, the difficulty in extracting titanium from ores, its high reactivity in the molten state, its forging complexity, its machining difficulty, and its sensitivity to segregation and inclusions required the development of special new design processing methods [4]. 
A wider adoption of titanium in a broader range of applications warranted by the material properties is hindered by high costs. Piecewise technological improvements are required to enable more widespread titanium usage. Many individual aspects of the process of making titanium components have peaked regarding performance. The production of converting titanium ore into titanium sponge is accomplished using the Kroll process [5]. This involves the high-temperature reaction of chlorine with titanium oxide, in the form of rutile or ilmenite, to form titanium tetrachloride. In the next step, Magnesium is reduced with the titanium tetrachloride to form magnesium chloride and elemental titanium. At this point, the titanium can be separated from the magnesium chloride for use. The downside of this process is the energy requirements, as titanium requires more energy to refine than all other structural metals [6].

The development of the titanium industry can be classified into two periods. The first period lies between the 1950s to the mid-1980s, and this constituted a period of intense progress around the development of technology and processes around titanium. The second period from the mid-1980s to the present has been centered around a transformation to a commercial industry. In this transformation, the cost is now the dominant consideration toward usage [7]. The shift toward cost optimization has fallen short of enabling large-scale industrial adoption of titanium, despite its abundance and material properties. The demand for titanium is tied to a select group of industries, of which the aerospace industry is the most prominent.

Due to the severe limitation that high-cost places on the material, titanium consumption exhibits a strong cyclic nature, tied to global events in the aerospace industry [8]. In the current raw material supply situation, sponge production is operating at 85 to $90 \%$ of capacity. This situation is constantly changing, and it is expected that this is the crest of a boom cycle in production levels. Continuous turbulence in the supply and demand environment, justifying capacity expansions is increasingly tenuous. What is needed within the industry is a high volume consumer with a steady demand to expand the global market. Hindered by persistent high costs, titanium has been unable to escape its niche status in a few markets into other markets like automotive and consumer products that could grow the adoption of this structural metal [8].

Some small but burgeoning exceptions lie in biomedical use and additive manufacturing. Titanium alloys, because of their high strength-to-weight ratio and excellent corrosion resistance, have been widely used in biomedical implants [9-11]. Additive manufacturing (AM) is a desirable method for titanium alloy parts' fabrication, as it provides a low waste alternative for complex geometries [12]. However, these represent optimizations of single aspects of a supply chain or application. A top level view of optimization enables overall performance improvement while accounting for any detrimental implications within the system.

In this paper, the use of a system of systems' (SoS) design approach was used due to its capability to address complex multi-system integration problems associated with titanium metalworking and manufacture. A SoS is the composition of systems interacting, interrelated and interdependent components that form a complex and unified whole to meet SoS needs. The system of systems design approach, commonly employed in complex defense networks, was adapted and applied to address this manufacturing system problem.

\section{Materials and Methods}

\subsection{Materials}

Based on the lack of development with the production of titanium material (Ti-6Al-4V), the pursuit of net shape components will have one of the most profound impacts on component cost [13]. The more efficient utilization of titanium will have a significant role in the improvement in titanium component costs given the high raw material costs [14].

The cost of input materials and machining operations comprises approximately $67 \%$ of a finished component cost [15]. 
A net shape component would minimize both raw material and machining aspects of finished component costs and have a significant impact on finished component costs. Since forging costs are typically around $7 \%$, a marginal increase from changes in the forging process would not have as significant of an increase as reducing machining and reducing input materials. The system of systems design practices applied to the manufacturing supply chain will attempt to improve many of the low performing aspects of manufacturing components from titanium. In this analysis, costs associated with material usage are of primary consideration.

The main objective of this paper is to develop a cumulative method of producing net shaped components via existing metalworking technologies, rather than developing a new individual system of titanium production. To achieve this, the design of a supply chain will more thoroughly be analyzed to remove boundaries for achieving a net shape component. The proposed design methodology in this paper is commonly employed in defense networks to integrate many individual constitutive together to behave as one unified defense network. In these types of systems, the performance of the collective network is of the same importance as the individual constituent system. This method is referred to as the System of Systems (SoS) approach. This method is ideally suited to tailoring complex networks of systems to meet an overarching set of requirements [16]. In this way, the tendency to focus on individual systems and their requirements is avoided and the system of system performance is emphasized for the entire network or SoS. Though this technique is not commonly used in manufacturing supply chain analysis, it was observed by the lead author that supply chains experience similar complexities and interactions as integrated defense networks. To address the highly complex supply chain design problems, the system of systems framework was applied to a titanium supply chain to overcome the shortcomings in making components from titanium.

\subsection{Methods}

In an effort to accomplish any breakthrough in titanium component costs, the entire supply chain for manufacturing should be evaluated simultaneously. Due to the complex interaction of the many facets of the systems in a manufacturing supply chain, it is inferred that the supply chain in its entirety must be the focus of the design activity in order to be successful. Design improvements on a single facet of manufacture may have little to no effect on the manufacture of the component. If the improvement has a detrimental impact on another system in the supply chain, overall performance may be lowered. A system of system's design approach was used due to its capability to address complex multi-system integration problems [16].

In the department of defense, rapidly evolving requirements and acquisition methods have driven using "user capability needs" to develop new systems. Decisions have to be made from the broader capability portfolio context. Changing situational needs have driven the application of suites of systems or "System of Systems" to address the broader requirements [16]. This design approach was applied by the lead author to the industrial systems associated with titanium metalworking and manufacture to address the broader general problem of producing complex net-shape titanium components [17-19].

SoS are typically composed of many different systems at varying levels of maturity and development. These can include legacy systems, upgraded, and new systems. The systems engineer must make a system of systems level decision based on overall SoS needs and account for the unique considerations of each system simultaneously [16]. This is unlike the design of an individual system where the boundaries of an individual system represent the extent of development and envelop the system in a static manner. In SoS, the combined overall performance must be considered and accounted for. There is also a possibility for dynamic interaction or evolution in SoS structure that would not be present in traditional systems design. Table 1 illustrates the breakdown of SoS design activities involved in SoS design. The tasks identified as technical processes shown in Table 1 encompass the physical design aspects of designing a SoS. These tasks will form the sequence of tasks executed to drive SoS level design to improve a titanium manufacturing supply chain. 
Often, the first step in a SoS design is to generate an operational view (OV-1) for the SoS. This represents the environment of primary interactions that the SoS will encounter [16]. Figure 1 illustrates the OV-1 for a titanium manufacturing supply chain [16].

The system of systems under investigation is the supply chain section that produces finished structural titanium components from primary mill products, as illustrated above. That is not an indication that there are not gains to be made in the other aspects of titanium production, but merely a focusing on the consumption portion of the overarching titanium industry.

Table 1. System Engineering (SE) technical process applied to core SE elements [16].

\begin{tabular}{|c|c|c|c|c|c|c|c|}
\hline \multirow[b]{2}{*}{ Core SoS Elements } & \multicolumn{7}{|c|}{ Technical Design Processes } \\
\hline & 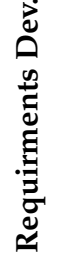 & 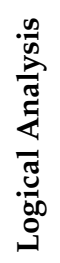 & 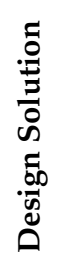 & 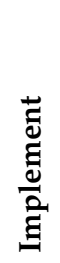 & 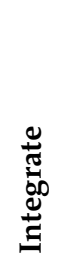 & $\stackrel{D}{D}$ & 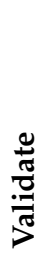 \\
\hline Translating Capability Objective & $X$ & & & & & & \\
\hline Understanding Systems \& Relationships & & $X$ & & & & & \\
\hline Assessing Performance to Capability Objectives & & & & & & & $\mathrm{X}$ \\
\hline $\begin{array}{l}\text { Developing and Evolving on SoS Architecture } \\
\text { Monitoring and Assessing Changes }\end{array}$ & $X$ & $x$ & $x$ & & & & \\
\hline Addresing Requirements and Solution Options & $X$ & & $X$ & & & & \\
\hline Orchestrating Upgrades & & & & $x$ & $\mathrm{X}$ & $X$ & $\mathrm{X}$ \\
\hline
\end{tabular}

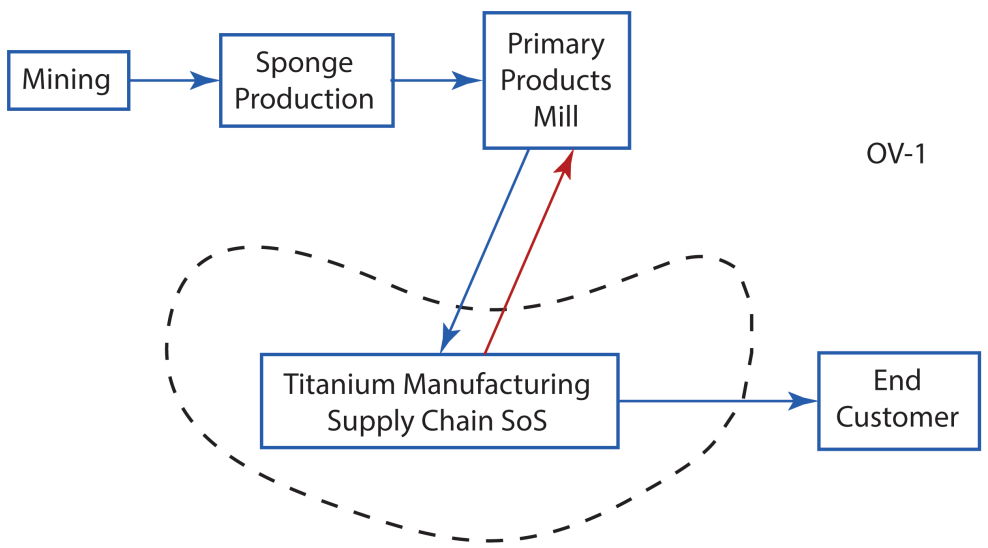

Figure 1. OV-1 for the supply chain of interest for manufacturing finished components from Wrought titanium material.

\section{Results and Discussions}

\subsection{Translating Capability Objectives}

The first core element of the system of systems design is the translation of system capabilities into SoS level requirements (see Table 1). The systems engineers are tasked with understanding and articulating technical level expectations for the cumulative system of systems. This forms the foundation and context for technical planning and capability evolution [16].

\subsubsection{Requirements Development}

As seen in Table 1, requirements development is the initial task in SoS design. At the highest level, the requirement of the system of systems is to produce a structural finished titanium component from 
a raw material or mill products. This is the highest level requirement of the system, as illustrated in Figure 2.

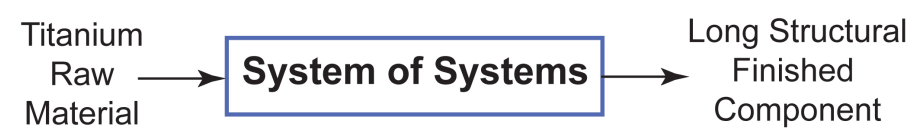

Figure 2. Highest level input and output of the system of systems under review.

In this situation, the SoS is a titanium metalworking supply chain with varying metalworking capabilities. The requirements for the supply chain as a whole are driven predominantly by the properties of the final product as perceived by the end user or customer. Since the end product is produced commonly today, many typical requirements can be gathered from industry specifications and open literature. Requirements governing a finished component are expected to fall within categories of process requirements, material property requirements, and end condition requirements. Process requirements are derived from customer expectations of a process performance. These expectations are to ensure that the system of systems performs in the interest of the end user or other stakeholders.

\section{Process Requirements}

Process requirements include unit cost, material efficiency and being environmentally responsible. These requirements are typically not stated in ranges but it is sought to maximize or minimize them out of the business interest of the end user. Unit cost is obviously one of the key metrics of manufacturing systems assuming all other aspects are equal. There are some reasons that material efficiency is a requirement for titanium manufacturing systems. The high cost and limited availability of titanium raw material is one of the primary drivers [6,8]. Environmental responsibility is an increasingly focused upon metric of industry [20].

\section{Material Properties Requirements}

The majority of requirements regarding material performance are based on the SAE Aerospace specification SAE AMS 4935 for titanium extrusion which is a common specification for long wrought products. These requirements include high strength, ductile, isotropic, homogeneity, chemistry, hydrogen concentration. These requirements are intended to characterize titanium's performance as is typically desired for industrial consumers. These requirements, though not all encompassing, are routinely tested for material components made from titanium from a variety of methods today [21].

\section{Condition Requirements}

The condition requirements are performance requirements of a finished component that also affect the physical performance of titanium. These include many of the characteristics of a typical finished component, which do not pertain to the material. These condition requirements include roughness, Alpha Case/Oxide free surface, free of any surface cracks, free of surface defects, complex shapes, and dimensional control. Within these requirements are some conditions that are simply not permissible in any structurally sound component such as the presence of cracks, surface defects or surface oxides. Others represent aspects typical for structural components, such as smooth surfaces, dimensional consistency, and complexity of form. These metrics were selected to represent the interest of a customer when evaluating a titanium structural component. These requirements were assembled from known performances of titanium as well as open literature about various aspects of production. 


\subsection{Understanding Systems Relationships}

\subsubsection{Logical Analysis}

As shown in Table 1, identification of Logical Analysis is the second task in SoS Design. When evaluating functionality required for the manufacturing system of systems, three predominant functions arise: preforming, material conditioning, and finishing. The conditional requirements around part complexity, surface condition, and dimensional control dictate the need for a finishing step to add component features and remove surfaces that are unsuitable for the finished component. The material properties requirements associated with finished components are typically conditioned to achieve these requirements. Most commonly for titanium, this condition is a hot working process coupled with a subsequent heat treatment process [21]. Finally, the process requirements for a low unit cost and high material efficiency dictate a preforming step to convert the raw mill stock into a form closer to the finished component, or "near net" preforming.

\section{Outline of SoS Functional Systems}

The system of systems for manufacturing titanium components should follow the general outline shown below. An initial process takes raw material which may or may not be the proper size to yield the desired component, called a preform in this work. Typically, the material would need some heat treatment to evoke the desired properties of the component. This is most commonly done before final steps to prevent adverse effects on a final component that are possible during heat treatment. The final functional system is the finishing step, which most commonly is a machining process, to extract the desired component from the preform and deliver many of the conditional requirements of the component such as surface finish, size, thickness, etc. Even though familiarity may lead one toward common systems, it may result in the elimination of possibly valid SoS architectures without consideration. In Figure 3, the functional mapping of the SoS is given. Heat treatment is specified because many industrial specifications dictate some form of heat treatment. It was therefore considered unyielding. If later analysis indicated it as being the vehicle for improvement, it could be challenged.

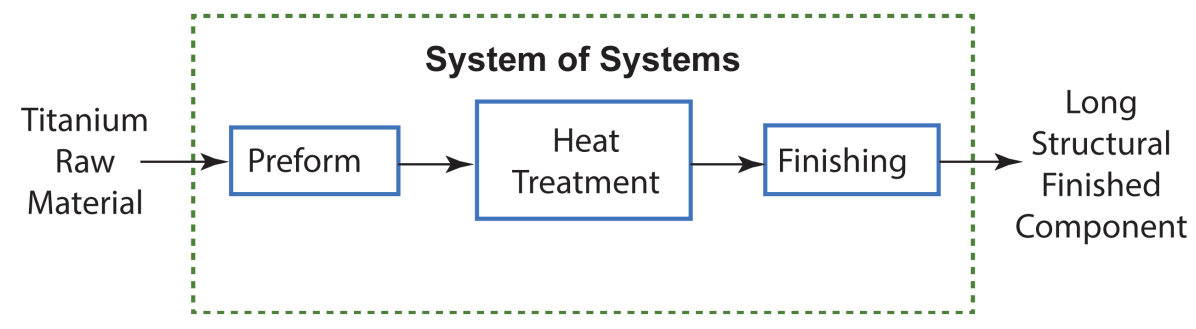

Figure 3. Conversion of Titanium raw material to structural beam titanium components.

System Influence on SoS Requirements

The identification of how the individual systems contribute to the SoS performance begins with the mapping of the systems to the specific requirements on which they have an impact, as shown in Figure 4.

Some clear relationships exist in the SoS. Clearly, most material property requirements arise from the preform and heat treatment process. Additionally, many of the conditional requirements that govern the component's form arise from finishing. One key relationship lies in that the material efficiency is defined or dictated by the preform process alone. The background information supporting this diagram is covered in more detail in the subsequent section. 


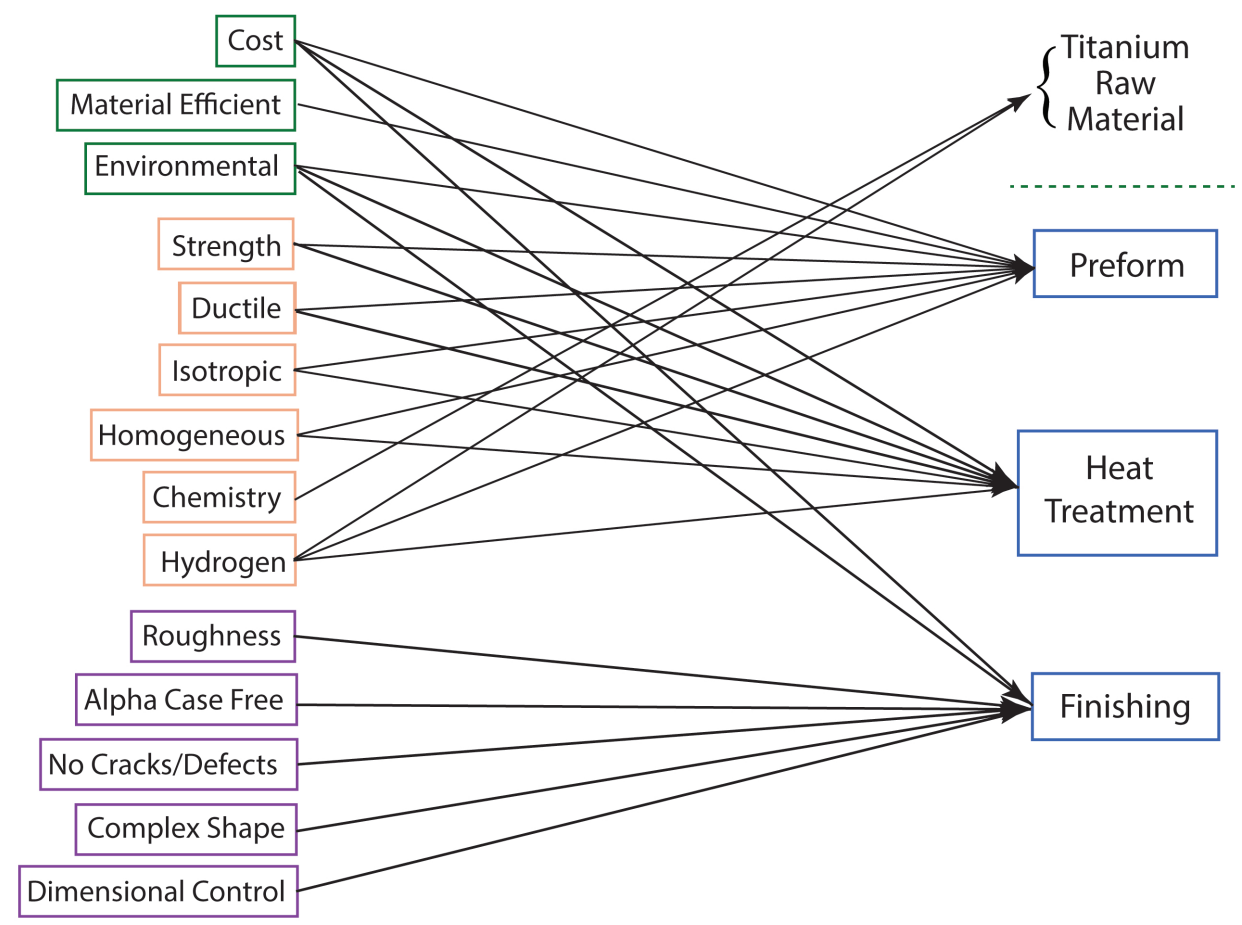

Figure 4. Mapping of the functional system in the SoS to the identified SoS requirements that govern the performance of the SoS.

System Interdependencies in Relation to SoS Requirements

Unit Cost: The understanding of cost is essentially a flow of money through the system of systems. A mapping of the functional SoS' impact on the cost requirement was performed and is illustrated in Figure 5. Black lines represent money flow, and red lines represent the flow of information.

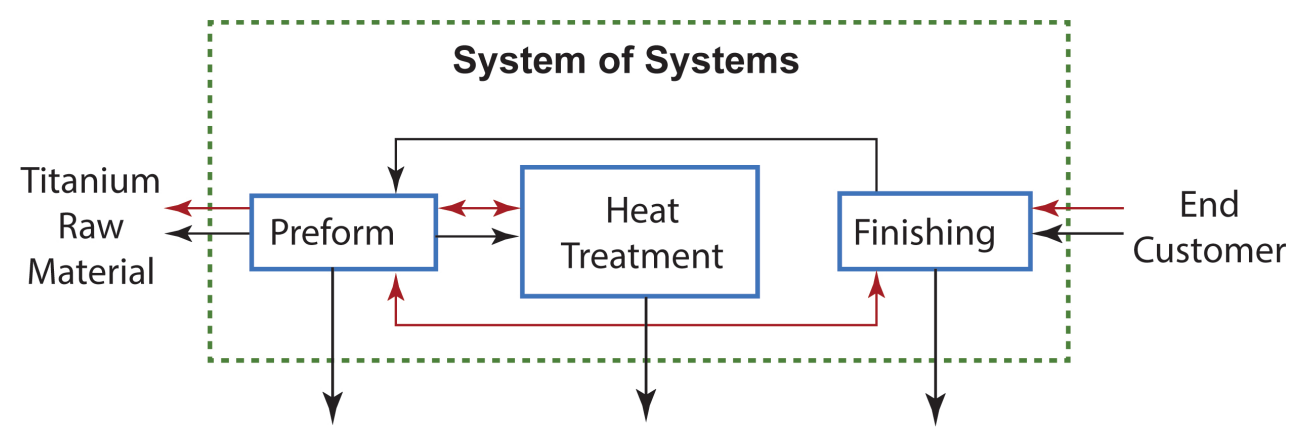

Figure 5. Flow of money through the system of systems.

It can be seen in the visualization above that the primary source of money is from the end customer in the form of payment for the product. The finishing function has an associated cost and profit allotment as well as payment for the input to that functional system. Based on the fact that in many of the industry specifications, the material properties are listed within specifications that govern the preform process, it is reasonable to assume that the heat treatment is being coordinated by the preform processer [21]. Heat treatment is commonly performed as a service as they cannot answer for the material they receive for processing. In the diagram, therefore, the information to the heat treatment system is illustrated as coming from the preform system. Heat-treated product would need to be tested before the preformer to be able to sell the product. This would indicate that the product for finishing would be purchased from the preformer. Due to this, the flow of money is assumed to circumvent the heat treatment step. The money for heat treatment would flow from the preform system instead. In the preform step, a portion of the money would be used for the cost 
and profitability of that functional system. The next in the chain would be the heat treatment process. Again, the allotment for cost and profitability is consumed. The remainder of the currency is allotted to purchase raw material from outside the system of systems.

Information flows through the system of systems in a similar direction. Given that it is believed that finishing and preform would immediately become cooperators, the information is expected to flow that way as well. The information pertinent to heat treatment and raw material would then come from the preform step.

Material Efficiency: The efficient utilization of material in producing components from titanium is one of the most significant measures of a successful titanium supply chain. Improvements in material efficiency are highlighted as having the largest impact on underlying cost pressures in the titanium industry [14]. The use of the perform operation, such as an extrusion operation, is intended primarily for this purpose. The operation in itself is intended to convert the raw material form into something that more efficiently encompasses the desired component. In this way, significant improvements can be made in contrast to using a solid bar input stock [22]. In most instances, the finishing step is represented by a machining process. The machining, or another subtractive process, is usually the predominant source of material loss in all machined component supply chains. By the definition of a subtractive process, it removes all material that does not represent the finished component. In forgings, for example, this constitutes upwards of $95 \%$ of titanium material input into the machining step [7].

Environmental Responsibility: The industry is becoming aware of the impacts of their operations on the environment. Awareness of environmental influence increasingly factors into decision making around manufacturing and increasing efforts to improve industrial practice are continuously pursued [20].

The production of titanium raw material is an extremely energy intensive process. Of all the widely used structural metals, titanium is the most resource-intensive to produce. Titanium represents $50 \%$ more energy consumption to produce than aluminum per pound and over 15 times that of steel [6]. This is unlikely to change in the immediate future as the Kroll process for producing titanium has been largely optimized. Currently, no immediate alternatives have been successfully scaled up from laboratory trials [8]. This is considered in the analysis of the system of systems because the amount of material purchased drives a significant source of environmental impact. As such, this is considered a direct attribute of the system of systems, though it arises outside of the SoS.

Energy consumption in thermomechanical deformation also contributes to environmental impact. To get enough ductility to accommodate significant amounts of deformation input, the preform must be worked at some elevated temperature [23]. As a result, there is some required amount of energy input to heat the material. This is considered the primary energy consumption for the preform step and is captured as an environmental impact. The material characteristics derived from the preform process also influence the impact of both the heat treatment process downstream and finishing process. The more excess material that is present in the near net shape component, the more mass must be heat treated and excess stock must be removed in the finishing system. The finishing system, such as machining, is typically energy intensive. It is estimated that just under 37,000 BTU are required per pound of titanium that is removed in the milling process [24]. This is a significant source of energy consumption and environmental impact.

Strength/Ductility/Hydrogen: The contributors to the strength and ductility of the material are going to be significantly impacted by a number of factors that pertain to the thermo-mechanical history of the material. The primary separation of different hot working processes is whether they are conducted above or below the beta transition (transus) temperature. In working above the beta transus, grain growth rates are significant, so time above the beta transus will strongly correlate with grain size. Beyond that, the other important attributes are the temperature, strain input, strain rates of input, chemistry, and starting microstructures [23]. The grain growth and change in microstructure would undoubtedly impact the strength and ductility in a negative way. 
When titanium is heated to an elevated temperature, it is common for hydrogen to go into solution to the bulk material. Unlike oxygen and nitrogen which readily react with titanium to form an outer layer, hydrogen permeates through the material [22]. The amount of hydrogen absorbed is a function of time and temperature history through the system of systems. In excessive quantities, hydrides will form within the material and embrittlement will occur [22]. A final parameter associated with heat treatment is the pressure associated with the heat treatment: performing heat treatment in a vacuum [16]. As a result, all associative hot working or elevated temperature steps are expected to impact the strength, ductility, and hydrogen levels of the system of system.

Isotropic/Homogeneous: Isotropy and homogeneity are key parameters when analyzing a titanium supply chain. These two parameters represent two of the most difficult aspects of working with titanium. Many preform processes that impart work in one direction, such as rolling or drawing, have the tendency to produce anisotropic material properties in titanium materials [25]. The temperature of rolling and level of unidirectional work have strong influences on the severity of anisotropy within a material [26]. There is a correlation between intermittent and subsequent heat treatments that can mitigate the amount of isotropy within the material [27]. This should be weighed in contrast to the impacts that additional heating will have on properties such as grain size and hydrogen levels $[23,28]$

Homogeneity is a key parameter of titanium material as well. Due to titanium's high flow softening and low thermal conductivity, a phenomenon called shear banding is common where non-uniform work is rapidly put into the material. In this work, temperature rise tends to concentrate non-uniformly into planes. When this happens, most work is performed along those planes while the intermittent material is not deformed [23]. This phenomenon is exaggerated at lower temperatures where flow stresses are higher [7]. The instance of heat loss to tooling also increases this effect [28]. This results in compromised bulk material. The concentration of deformation on the shear planes can produce strain-induced porosity and embrittlement along these planar structures [7]. Heat loss to tooling can also influence homogeneity by producing a layer of un-deformed material as a result of conduction to tooling [28]. The surface layer simply stiffens to the point that deformation is discouraged and accommodates elsewhere. Isotropy and homogeneity are driven by the preform and heat treatment systems.

Chemistry: The non-hydrogen chemical constituents of the material are not affected in all aspects of the proposed system of systems other than a subsequently removed surface layer. The chemistry is produced during the melting phases of the mill production. Chemistry targets result from the alloying phase of raw material production. Chemistry does have an impact on a variety of other parameters of the material such as strength and ductility. It is important that targets be achieved by the system of systems. The key functional behavior from the SoS is the information flow that directs proper raw material procurement.

Roughness/Surface Cracks and Defects/Dimensional Control: The roughness, dimensional control, and surface condition of a material have a significant impact on the performance of a final component. Fatigue, in particular, is subject to the condition of the surfaces, as imperfections serve as initiation points for crack growth through the material [29].

The preform function is one of the predominant drivers of the surface quality of a component. The surface layer resulting from the preform system dictates a number of aspects of the SoS, such as how much material the finishing system must remove. In a hypothetical extrusion preform step, the use of lubrication lowers the quality of extruded surfaces as compared to surfaces of other materials performed without lubrication [30]. However, without lubrication, titanium exhibits a high propensity for galling and seizing. This results in striations and tears in the surfaces [31]. In a hypothetical rolling preform, roughening would occur due to strain accommodations at the termination points of slip plains. This manifests in a very fine cracked-like appearance [32].

The finishing step is highly coupled to the preform operation in the system of systems. Much of the design work and communication facilitates coordination between systems to ensure that the output 
of the preform is what the finishing step requires to achieve removal of poor surfaces and delivery of dimensional performance. The finishing step also has an impact on these parameters independent of the preform function. Cutting the material too fast can result in galling, welding, or smearing of the metal surfaces. This can also result in tool failure.

Alpha Case: In all hot working and elevated temperature processes, there is potential to form a thin and highly alloyed layer called alpha case on the outer surface [33]. The growth of this layer is shown to increase markedly between $1061 \mathrm{~K}$ and $1172 \mathrm{~K}$ in oxygen-containing environments [34]. In the extrusion industry specification, a requirement for removal prior to delivery for machining exists [21]. This is due to the negative impact it has on machining and finishing tooling. The brittle layer has been shown to increase the incidence of spalling of bits at the beginning and end of a cut [22]. The finishing process can remove the alpha case if the negative effects on it are tolerable and removal amounts are sufficient. As a result, the requirement for removal from the finished component is a near certainty due to the negative impact on tensile ductility and fatigue resistance [34].

\subsection{Assessing Performance to Capability Objectives}

The function of assessing and comparing the performance of the SoS in comparison to developed capability objectives is key for formulating improvement plans in the system of systems. Metrics need to be established independently of system specifics to prevent self-guiding toward and answer. This has been done to this point. Through the performance assessment phase, specific areas of focus can be identified for improvement to benefit the SoS [16].

\subsubsection{Validation}

In the validation phase associated with assessing the performance of a system of systems in relation to capability objectives, the formation of performance metrics form the benchmark for which progression is undertaken. It is also important that metrics be ranked by importance as not all metrics have the same importance as others [16]. In the current supply chain, requirements are going to be ranked as tier 1 or tier 2 as per the system engineer's preference. Tier 1 requirements are of primary concern. These requirements represent a foremost metric by a customer that could be a significant differentiator of the system of systems. Tier 2 requirements represent conditions or performances that are expected to align with industry standards but do not define a differentiator. In other words, the tier 1 requirements will be those for which improvement is most sought. The Tier 2 requirements establish performances that must meet minimum standards or existing performance levels.

\section{Tier 1 Requirements}

Tier 1 requirements include unit cost and material efficiency. These requirements were selected based on customer feedback in the form of industry analysis. Unit cost is an echoing of a significant body of research highlighting high cost as a major inhibitor of the wider adoption of titanium into the industry [5]. Material efficiency also represents a paramount requirement for a titanium supply chain. Many of the process aspects of producing titanium have matured and been optimized [8]. Therefore, the efficient utilization of material is highlighted as the most significant avenue for improvement for titanium manufacturing [14]. Improvements in cost and material efficiency would separate one supply chain from another to key stakeholders, which is why they are Tier 1 requirements.

\section{Tier 2 Requirements}

Tier 2 requirements represent a performance that is important to a customer but it is not imperative that improvement is realized. What is imperative is that performance is at least preserved and improved if possible. These represent areas outside the main thrust of development. It is important to note that these can be shifted between tiers depending on the development objectives of systems engineers.

Tier 2 requirements include environmental responsibility, strength, ductility, isotropy, homogeneity, chemistry, hydrogen content, surface roughness, alpha case depth, surface cracking, 
complex shapes, and dimensional control. These Tier 2 requirements represent performance parameters that define the performance of a component produced by a titanium manufacturing SoS. They are therefore important to a shareholder of the SoS but do not necessarily require improvement to meet the needs of the customer. The material property requirements epitomize this for titanium SoS. The material properties of titanium are desirable today in comparison to other structural materials [7]. There is not necessarily a need to improve these aspects to serve an unmet need of a stakeholder. As a Tier 2 requirement, performance must be at least preserved. No objection would be made to improvement in performance, but the loss of performance would not be acceptable. Furthermore, the conditional requirements represent parameters of a component produced in a titanium manufacturing supply chain. It is therefore assumed that these requirements were placed due to explicit needs and must, therefore, be maintained to continue meeting the same customer need. Any loss in these metrics would compromise the implication that needs are still met.

To establish a benchmark, a validation of process capabilities must be undertaken to determine existing SoS performance to capability metrics. All revisions to the SoS can then be measured against the initial case, to determine the degree to which a change resulted in improvement of the SoS [8]. However, this is not the time to define what functional states exist for the final SoS; the selection of a functioning SoS with specific steps is essential to provide some performance baseline for any SoS that may result. The chosen existing supply chain SoS is centered around a titanium extrusion preform system and machining finishing system. This system is composed of five functional systems. The extrusion step is the primary conversion step to make a component from a billet produced by a mill. There is also a heat treatment, straightening, and chemical milling systems that are coupled with the extrusion process (see Figure 6). The collective of these functional systems is expected to deliver properties with requirements aligned with industry specification SAE AMS 4935, in some cases [21]. It is noteworthy that these systems are so commonly coupled with extrusion that they are listed within the same industry specification [21]. The collective of these systems defines much of the material property requirements of the final component as this encompasses the thermo-mechanical history of a component. The next and final functional system is the machining step. This removes material to produce the final component from the extrusion preform. This step generates much of the conditional requirement performance such as surface quality, surface defects, and thickness control.

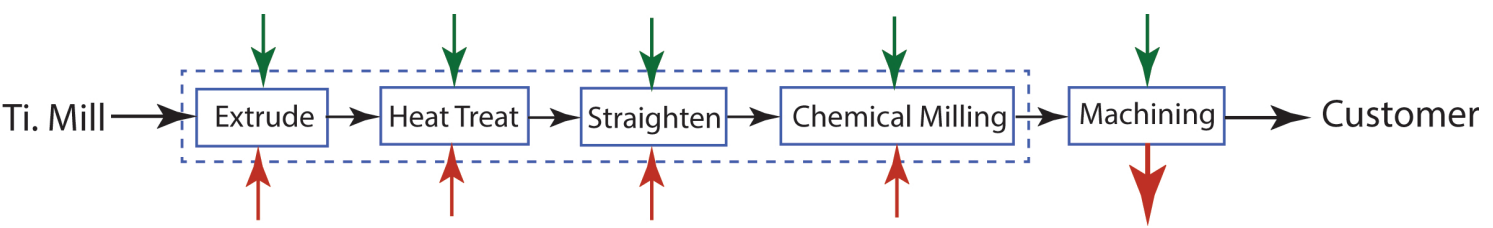

Figure 6. Skeleton of the baseline SoS to convert mill material into a finished titanium structural component.

\section{Conclusions}

The onset of this effort was to address the system's inadequacies at meeting demands from within various industries to deliver components more cost effectively. Such a broadly stated problem, involving a complex manufacturing network, requires a systematic approach in order to facilitate addressing the problem in the most succinct manner. The system of systems design approach, commonly employed in complex defense networks, was adapted and applied to address this manufacturing system problem.

As shown in Table 1, in the first part of this paper we discussed three "core SoS elements" in detail. We identified various methodological challenges which have to be addressed when designing proposed integrated SoS. The second part of the paper will cover the remaining core SoS elements and propose an integrated design for the titanium metalworking and manufacturing system of systems.

\section{Patents}

Patent application (Application US20170306467A1) has been filed by RTI International Metals, Inc. 
Author Contributions: This paper was produced from A.S.'s dissertation. A.E. was the advisor of A.S. A.E. wrote the paper using A.S.'s Ph.D. dissertation.

Funding: This research did not receive any specific research grant from funding agencies in the public, commercial, or not-for-profit sec-tors. Support of experimentation, including the use of equipment, was furnished by RTI International Metals, Inc.

Conflicts of Interest: The authors declare no conflict of interest.

\section{References}

1. Department of Mineral Resources, South Africa. An Overview of South Africa's Titanium Mineral Concentrate Industry. 2008. Available online: citeseerx.ist.psu.edu/viewdoc/download?doi=10.1.1.167. 7253\&rep=rep1\&type=pdf (accessed on 22 March 2019).

2. Józwik, J. Evaluation of Tribological Properties and Condition of Ti6Al4V TitaniumAlloy Surface. Tehnicki Vjesnik 2018, 25, 170-175.

3. Beranoagirre, A.; Urbikain, G.; Calleja, A.; López de Lacalle, L.N. Drilling Process in $\gamma$-TiAl Intermetallic Alloys. Materials 2018, 11, 2379. [CrossRef] [PubMed]

4. Beranoagirre, A.; López de Lacalle, L.N. Grinding of Gamma TiAl Intermetallic Alloys. Procedia Eng. 2013, 63, 489-498. [CrossRef]

5. Sibum, H. Titanium and Titanium Alloys-From raw material to semi-finished products. Adv. Eng. Mater. 2003, 5, 393-398. [CrossRef]

6. Norgate, T.E.; Jahanshahi, S.; Rankin, W.J. Assessing the Environmental Impact of Metal Production Processes. J. Clean. Prod. 2007, 15, 838-848. [CrossRef]

7. Lutjering, G.; Williams, J.C. Titanium, 2nd ed.; Springer: Berlin/Heidelberg, Germany; New York, NY, USA, 2003.

8. Jackson, M.; Dring, K. A review of advances in processing and metallurgy of titanium alloys. Mater. Sci. Technol. 2006, 22, 881-887. [CrossRef]

9. Cao, X.; Xu, X.; Wang, C.; Pyoun, Y.; Wang, Q. Effect of ultrasonic surface impact on the fatigue behavior of ti-6al-4v subject to simulated body fluid. Metals 2017, 7, 440. [CrossRef]

10. Du, J.-K.; Chao, C.-Y.; Chiu, K.-Y.; Chang, Y.-H.; Chen, K.-K.; Wu, J.-H.; Wu, J.-N. Antibacterial properties and corrosion resistance of the newly developed biomaterial, ti-12nb-1ag alloy. Metals 2017, 7, 566. [CrossRef]

11. Józwik, J.; Ostrowski, D.; Milczarczyk, R.; Krolczyk, M.G. Analysis of relation between the 3D printer laser beam power and the surface morphology properties in Ti-6Al-4V titanium alloy parts. J. Braz. Soc. Mech. Sci. Eng. 2018, 40, 1678-5878. [CrossRef]

12. Agius, D.; Kourousis, K.I.; Wallbrink, C. A review of the as-built slm ti-6al-4v mechanical properties towards achieving fatigue resistant designs. Metals 2018, 8, 75. [CrossRef]

13. Boyer, R.R.; Cotton, J.D.; Chellman, D.J. Titanium for Airframe Applications: Present Status and Future Trends. In Proceedings of the 10th World Conference on Titanium, Hamburg, Germany, 13-18 July 2003.

14. Schauerte, O. Titanium in automotive production. Adv. Eng. Mater. 2003, 5, 411-418. [CrossRef]

15. Sanders, D.; Boyer, R.R.; Cotton, J.; Watts, M. Titanium Manufacturing Processes and Alloy Selection for Aerospace Applications. In Proceedings of the International Titanium Association Conference, Atlanta, GA, USA, 7-10 October 2012. Available online: https:/ /cdn.ymaws.com/titanium.org/resource/resmgr/2010_ 2014_papers/SandersDaniel_2012.pdf (accessed on 28 February 2019).

16. Office of the Deputy Under Secretary of Defense for Acquisition and Technology, Systems, and Software Engineering. Systems Engineering Guide for Systems of Systems, version 1.0; ODUSD(A\&T) SSE: Washington, DC, USA, 2008.

17. Stroud, A.; Ertas, A. Development of a rolling finishing system to deliver net shape components from titanium structural extruded shapes. J. Manuf. Processes 2018, 32, 187-198. [CrossRef]

18. Stroud, A. Development of Net Shape Structural Titanium Manufacturing Capability Using the System of Systems Design Approach. Ph.D. Thesis, Mechanical Engineering Department, Texas Tech University, Lubbock, TX, USA, 2018.

19. Stroud, A. System of systems analyses of rti international metals' boeing 787 seat rail supply chain. Transdiscipl. J. Eng. Sci. 2014, 5, 35-51. [CrossRef] 
20. Berry, M.A.; Rondinelli, D.A. Proactive corporate environmental management: a new industrial revolution. Acad. Manag. Exec. 1998, 12, 38-50. [CrossRef]

21. Extrusions, T.A.; Rings, F.W. 6Al-4V, Annealed, Beta Processed. SAE AMS 4935K; AMS G Titanium and Refractory Metals Committee: Warrendale, PA, USA, 2014.

22. Froes, F.H. Titanium: Physical Metallurgy, Processing, and Applications; ASM International: Novelty, OH, USA, 2015.

23. Tamirisakandala, S.; Bhat, R.B.; Vedam, B.V. Recent advances in the deformation processing of titanium alloys. J. Mater. Eng. Perform. 2003, 12, 661-673. [CrossRef]

24. Rajemi, M.F.; Mativenga, P.T.; Aramcharoen, A. Sustainable machining: Selection of optimum turning conditions based on minimum energy considerations. J. Clean. Prod. 2010, 18, 1059-1065. [CrossRef]

25. Frederick, S.F.; Lenning, G.A. Producing basal textured Ti-6Al-4V sheet. Metall. Trans. B 1975, 6, 601-605. [CrossRef]

26. Amateau, M.F.; Dull, D.L.; Raymond, L. The effect of processing on plastic anisotropy of Ti-6Al-4V. Metall. Trans. 1974, 5, 561-564. [CrossRef]

27. Williams, J.C. Titanium and Titanium Alloys: Scientific and Technological Aspects; Springer Science \& Business Media: Berlin, Germany, 2013; Volume 3.

28. Semiatin, S.L.; Seetharaman, V.; Weiss, I. The thermomechanical processing of alpha/beta titanium alloys. J. Miner. Met. Mater. Soc. 1997, 49, 33-39. [CrossRef]

29. Froes, F.H.; Eylon, D.; Bomberger, H.B. Titanium Technology: Present Status and Future Trends; Titanium Development Association: Dayton, OH, USA, 1985; pp. 1-19.

30. Chadwick, R. The hot extrusion of non-ferrous metals. Int. Mater. Rev. 1959, 4, 189-256. [CrossRef]

31. Owens, R.S.; Roberts, R.W. Mechanism of Iodine Lubrication. Nature 1971, 229, 183-184. [CrossRef]

32. Ginzburg, V.B. Steel Rolling Technology: Theory and Practice; Marcel Dekker Inc.: New York, NY, USA, 1989.

33. Mitchell, A. Melting, Casting and Forging Problems in Titanium Alloys. Mater. Sci. Eng. A 1998, 243, $257-262$. [CrossRef]

34. Gurappa, I. Prediction of titanium alloy component life by developing an oxidation model. J. Mater. Sci. Lett. 2003, 22, 771-774. [CrossRef]

(C) 2019 by the authors. Licensee MDPI, Basel, Switzerland. This article is an open access article distributed under the terms and conditions of the Creative Commons Attribution (CC BY) license (http:/ / creativecommons.org/licenses/by/4.0/). 